\title{
Flavor symmetry breaking in the $\Delta$ sea
}

\author{
J. J. Ethier, ${ }^{1}$ W. Melnitchouk, ${ }^{2}$ Fernanda Steffens, ${ }^{3}$ and A. W. Thomas ${ }^{4}$ \\ ${ }^{1}$ Department of Physics and Astronomy, Vrije Universiteit Amsterdam, 1081 HV Amsterdam, \\ and Nikhef Theory Group, Science Park 105, 1098 XG Amsterdam, The Netherlands \\ ${ }^{2}$ Jefferson Lab, Newport News, Virginia 23606, USA \\ ${ }^{3}$ Institüt für Strahlen- und Kernphysik, Universität Bonn, 53115 Bonn, Germany \\ ${ }^{4}$ CSSM and ARC Centre of Excellence for Particle Physics at the Terascale, Department of Physics, \\ University of Adelaide, SA 5005, Australia
}

(Received 18 September 2018; published 12 August 2019)

\begin{abstract}
The discovery of a sizeable asymmetry in the $\bar{u}$ and $\bar{d}$ distributions in the proton was one of the more consequential experimental findings in hadron physics last century. Although widely believed to be related to the fundamental role of chiral symmetry in QCD, a definitive verification of this hypothesis has remained elusive. We propose a novel test of the role of chiral symmetry in generating the sea flavor asymmetry by comparing the $\bar{d}-\bar{u}$ content in the proton with that in the $\Delta^{+}$baryon, where a significant enhancement is expected around the opening of the $N \pi$ decay channel. Recent developments in lattice QCD suggest a promising way to test this prediction in the near future.
\end{abstract}

DOI: 10.1103/PhysRevD.100.034014

As a result of considerable theoretical and experimental effort, we now know that the sea of quark-antiquark pairs in the nucleon is far more complex than originally envisaged on the basis of simple quark models or perturbative QCD. The first major surprise was the confirmation in the early 1990s of an integrated excess of $\bar{d}$ over $\bar{u}$ antiquarks in the proton [1], leading to a violation of the Gottfried sum rule [2]. Almost a decade earlier, as a by-product of a study of the excess of nonstrange over strange sea quarks predicted within the cloudy bag model $[3,4]$, it had been shown that the application of chiral symmetry to the structure of the nucleon naturally led to a surplus of $\bar{d}$ over $\bar{u}$ [5].

Once the experimental result was announced, a number of calculations confirmed that the pion cloud picture could indeed explain it quantitatively [6-9]. Furthermore, a careful study of the nonanalytic behavior of the sea quarks as a function of quark mass established that the pion cloud contribution was an essential feature of spontaneous symmetry breaking in QCD [10-14]. Studies of the sea using Drell-Yan lepton-pair production [15] in $p \bar{p}$ collisions at Fermilab suggested an unexpected change of sign in $\bar{d}-\bar{u}$ at parton momentum fractions $x$ around 0.3 [16], which is difficult to accommodate naturally within a meson cloud framework [17]. While we await the results of the

Published by the American Physical Society under the terms of the Creative Commons Attribution 4.0 International license. Further distribution of this work must maintain attribution to the author(s) and the published article's title, journal citation, and DOI. Funded by SCOAP ${ }^{3}$. follow-up SeaQuest experiment [18], designed to explore the asymmetry to larger $x$, it is imperative to obtain independent confirmation of the physical mechanism.

Here we suggest that a comparison of the $\bar{d}-\bar{u}$ asymmetry in the $\Delta^{+}$baryon with that in the proton provides an outstanding opportunity for such a confirmation. To understand why, we recall that the dominant meson-baryon component of the proton wave function arises from quantum fluctuation $p \rightarrow n \pi^{+}$. As the $\pi^{+}$contains only a valence $\bar{d}$ antiquark, one naturally expects $\bar{d}>\bar{u}$ in the proton. The process $p \rightarrow p \pi^{0}$, which is suppressed by a factor of 2 by isospin couplings, produces equal numbers of $\bar{d}$ and $\bar{u}$ and therefore does not affect the asymmetry. While the process $N \rightarrow \Delta \pi$ acts to reduce the asymmetry, it is suppressed relative to the dominant process $N \rightarrow N \pi$.

For the $\Delta^{+}$baryon, the processes $\Delta \rightarrow \Delta \pi$ and $\Delta \rightarrow N \pi$ both favor $\pi^{+}$production, and hence also produce an excess of $\bar{d}$ over $\bar{u}$. The key difference, however, is that because the $\Delta$ decay to $N \pi$ is favored energetically, it experiences a significant kinematical enhancement as a function of the pion mass, $m_{\pi}$, as it approaches the $\Delta-N$ mass difference and the decay channel opens up.

In parallel developments, recent progress in the calculation of parton distribution functions (PDFs) in lattice QCD suggests a realistic means to check the prediction. In particular, lattice QCD measurement of the spatial correlation function of quarks within a fast moving hadron could be used [19], after Fourier transformation and renormalization, to obtain a quasi-PDF [20-22]. Through a further matching procedure [23-25], one can then directly obtain the desired light-cone PDF over the range $x \in(-1,+1)$. 
Previous attempts to extract antiquark distributions from lattice QCD were impaired by the difficulty of disentangling the $q$ and $\bar{q}$ content using only the first two or three moments from calculations of matrix elements of local twist-two operators [26]. In contrast, in the quasi-PDF approach, one can use the crossing symmetry relation, $\bar{q}(x)=-q(-x)$, to extract directly the $x$ dependence of the $\bar{q}$ PDFs. Exploratory studies of quasi-PDFs [24,27-29] did indeed suggest an asymmetric sea, even though renormalization was not yet available, and the computations were performed at large pion masses.

Recently, however, simulations at the physical pion mass, including a sophisticated treatment of renormalization, have shown a promising degree of agreement with empirical distributions [30,31]. Nonetheless, a number of systematics, such as discretization and finite volume effects, as well as difficulties in dealing with high momentum hadrons on the lattice, have to be addressed before quantitative comparisons with phenomenology are possible. In this spirit, a measurement of the distribution $u-d$ in the $\Delta^{+}$would be of enormous interest, especially if the difference between the $u-d$ shapes in the $\Delta^{+}$and proton were to be sufficiently large compared to the computational uncertainties.

Within a chiral effective theory framework, the asymmetry between the $\bar{d}$ and $\bar{u}$ PDFs in a baryon $B(B=N$ or $\Delta$ ) arises through a convolution of the valence antiquark distribution in the pion, $\bar{q}_{v}^{\pi}$, and the corresponding lightcone momentum distribution, $f_{B \rightarrow B^{\prime} \pi}$, of pions in $B$ with a spectator baryon $B^{\prime}$ [14,32-35]. The coupling of the external probe to the pion field in the effective theory arises through the rainbow diagrams illustrated in Fig. 1, as well as via bubble diagrams in which the pion loop couples to the baryon $B$ via a Weinberg-Tomazawa four-point interaction $[13,14,32-35]$. The latter involves pions with zero momentum fractions $y$ and are localized to $x=0$. Since lattice QCD simulations cannot access PDFs at $x=0$, the bubble diagrams will not be relevant here.

Moreover, the rainbow diagrams themselves receive zero mode contributions [14,34], in addition to the usual on-shell terms at $x>0$. Off-shell and Kroll-Ruderman

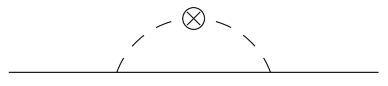

(a)

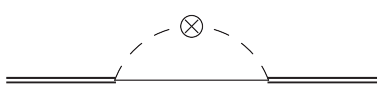

(c)

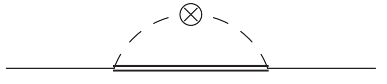

(b)

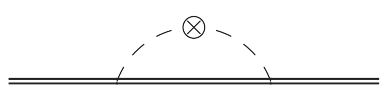

(d)
FIG. 1. Pion loop diagrams contributing to the $\bar{d}-\bar{u}$ PDFs in the nucleon (solid lines) and $\Delta$ (double solid lines) from the processes (a) $N \rightarrow N \pi$, (b) $N \rightarrow \Delta \pi$, (c) $\Delta \rightarrow N \pi$, and (d) $\Delta \rightarrow \Delta \pi$, with the $\otimes$ representing the insertion of a nonlocal current operator. terms contribute to the quark distributions through coupling to the intermediate state baryon $B^{\prime}[32,35]$. The dominant contributions to the antiquark asymmetry in the proton and $\Delta^{+}$at $x>0$ are then given by

$$
(\bar{d}-\bar{u})^{p}(x)=2\left[\left(f_{N \rightarrow N \pi}-f_{N \rightarrow \Delta \pi}\right) \otimes \bar{q}_{v}^{\pi}\right](x),
$$

and

$$
(\bar{d}-\bar{u})^{\Delta^{+}}(x)=\left[\left(f_{\Delta \rightarrow N \pi}+2 f_{\Delta \rightarrow \Delta \pi}\right) \otimes \bar{q}_{v}^{\pi}\right](x),
$$

where the symbol " $\otimes$ " denotes the convolution operator

$$
[f \otimes g](x) \equiv \int_{x}^{1} \frac{d y}{y} f(y) g\left(\frac{x}{y}\right) .
$$

The expressions for the splitting functions appearing in Eqs. (1) and (2) are ultraviolet divergent and must be regularized. In the literature, various regularization schemes have been advocated, including transverse momentum cutoff, Pauli-Villars, and dimensional regularization (DR), as well as form factors or finite-range regulators [9,32,33,35-38]. Within DR, specific power counting schemes can be preserved in the formal chiral perturbation theory expansions. On the other hand, finiterange regulators account for the finite size of hadrons, effectively resumming terms in the chiral series [36]. In practice, this allows for better convergence in $m_{\pi}$ in regions where the usual power counting schemes would not otherwise be applicable [39,40]. Following Ref. [41], we use a dipole form factor with cutoff parameter $\Lambda$ to regulate the UV divergences,

$$
F(k)=\left(\frac{\bar{\Lambda}^{2}}{\Lambda^{2}-k^{2}}\right)^{2},
$$

where $\bar{\Lambda}^{2} \equiv \Lambda^{2}-m_{\pi}^{2}$, so that the form factor is normalized to unity at the pion pole.

For a proton target, the $N \rightarrow N \pi$ splitting function for Fig. 1(a) at $y>0$ is then given by the familiar on-shell contribution $[5,34,41,42], f_{N \rightarrow N \pi}(y>0)=f_{N \rightarrow N \pi}^{(\text {on })}(y)$, where

$$
f_{N \rightarrow N \pi}^{(\mathrm{on})}(y)=\frac{g_{A}^{2} M^{2} \bar{\Lambda}^{8}}{\left(4 \pi f_{\pi}\right)^{2}} \int d k_{\perp}^{2} \frac{y\left(k_{\perp}^{2}+y^{2} M^{2}\right)}{\bar{y}^{2} D_{N N}^{2} \tilde{D}_{N N}^{2}},
$$

with $g_{A}$ the nucleon axial charge, $f_{\pi}$ the pion decay constant, $M$ the nucleon mass, $k_{\perp}$ the transverse momentum of the pion, and we define $\bar{y} \equiv 1-y$. The function $D_{N N}$ is the pion virtuality $k^{2}-m_{\pi}^{2}$, which in general depends on the initial and final state baryon masses, $M_{B}$ and $M_{B^{\prime}}$, respectively,

$$
D_{B B^{\prime}}=-\frac{1}{\bar{y}}\left[k_{\perp}^{2}-y \bar{y} M_{B}^{2}+y M_{B^{\prime}}^{2}+\bar{y} m_{\pi}^{2}\right],
$$


and the cutoff dependent factor $\tilde{D}_{N N}$ corresponds to Eq. (6) but with $m_{\pi}^{2}$ replaced by the cutoff parameter $\Lambda^{2}$,

$$
\tilde{D}_{B B^{\prime}}=-\frac{1}{\bar{y}}\left[k_{\perp}^{2}-y \bar{y} M_{B}^{2}+y M_{B^{\prime}}^{2}+\bar{y} \Lambda^{2}\right] .
$$

For the corresponding process $N \rightarrow \Delta \pi$ in Fig. 1(b), the splitting function at $y>0$ is given by a sum of on-shell and end-point contributions [33,41], $f_{N \rightarrow \Delta \pi}(y>0)=f_{N \rightarrow \Delta \pi}^{(\text {on })}(y)+f_{N \rightarrow \Delta \pi}^{\text {(end) }}(y)$,

$$
\begin{aligned}
& f_{N \rightarrow \Delta \pi}^{\text {(on })}(y)=\frac{g_{A}^{2} \bar{\Lambda}^{8}}{25 M_{\Delta}^{2}\left(4 \pi f_{\pi}\right)^{2}} \int d k_{\perp}^{2} \frac{y\left(\bar{M}^{2}-m_{\pi}^{2}\right)}{\bar{y}}\left[\frac{\left(\bar{M}^{2}-m_{\pi}^{2}\right)\left(\Delta^{2}-m_{\pi}^{2}\right)}{D_{N \Delta}^{2} \tilde{D}_{N \Delta}^{4}}-\frac{\bar{M}^{2}-3 m_{\pi}^{2}+2 \Delta^{2}}{D_{N \Delta} \tilde{D}_{N \Delta}^{4}}\right], \\
& f_{N \rightarrow \Delta \pi}^{\text {(end })}(y)=\frac{g_{A}^{2} \bar{\Lambda}^{8}}{25 M_{\Delta}^{2}\left(4 \pi f_{\pi}\right)^{2}} \int d k_{\perp}^{2} \frac{y}{\bar{y}^{2}}\left[\frac{k_{\perp}^{2}+y^{2} M^{2}-2 y\left(\bar{M}^{2}-M \Delta\right)-2 \bar{y} m_{\pi}^{2}+3 \bar{M}^{2}-4 M M_{\Delta}}{\tilde{D}_{N \Delta}^{4}}\right],
\end{aligned}
$$

where $M_{\Delta}$ is the $\Delta$ mass, and we have defined $\bar{M} \equiv M+M_{\Delta}$ and $\Delta \equiv M_{\Delta}-M$. In the $\Lambda \rightarrow \infty$ limit, the end-point term becomes a $\delta$-function at the kinematical end point, $y=1$, but for finite $\Lambda$, it remains finite at $y<1$. In this case, the sum of the on-shell and end-point contributions reduces to the simple result for $y>0$ [17],

$$
f_{N \rightarrow \Delta \pi}(y)=\frac{g_{A}^{2} \bar{\Lambda}^{8}}{25 M_{\Delta}^{2}\left(4 \pi f_{\pi}\right)^{2}} \int d k_{\perp}^{2} \frac{y\left[k_{\perp}^{2}+\left(M_{\Delta}-\bar{y} M\right)^{2}\right]\left[k_{\perp}^{2}+\left(M_{\Delta}+\bar{y} M\right)^{2}\right]^{2}}{\bar{y}^{2} D_{N \Delta}^{2} \tilde{D}_{N \Delta}^{4}} .
$$

In the chiral limit, moments of the splitting functions can be expanded in power series in $m_{\pi}$, with the leading nonanalytic terms in the expansion, which depend only on the long-distance properties of pion loops, being model independent [43]. For the $N \rightarrow N \pi$ distribution, one finds the characteristic leading order (LO) $\sim m_{\pi}^{2} \log m_{\pi}^{2}$ nonanalytic behavior [10-14]. Moments of the $N \rightarrow \Delta \pi$ splitting function, in contrast, display the next-to-leading order (NLO) behavior $\sim m_{\pi}^{4} \log m_{\pi}^{2}$ for $m_{\pi} \rightarrow 0[10,13,32,33]$.

In the case of a $\Delta$ baryon initial state, the on-shell and end-point contributions to the LO diagonal $\Delta \rightarrow \Delta \pi$ splitting function in Fig. 1(d) are given by

$$
\begin{aligned}
& f_{\Delta \rightarrow \Delta \pi}^{(\text {on })}(y)=\frac{g_{A}^{2} \bar{\Lambda}^{8}}{50 M_{\Delta}^{2}\left(4 \pi f_{\pi}\right)^{2}} \int d k_{\perp}^{2} \frac{y}{\bar{y}^{2}}\left[\frac{m_{\pi}^{2}\left[m_{\pi}^{2}\left(2 M_{\Delta}^{2}-m_{\pi}^{2}\right)-10 M_{\Delta}^{4}\right]}{D_{\Delta \Delta}^{2} \tilde{D}_{\Delta \Delta}^{4}}+\frac{\left.m_{\pi}^{2}\left(4 M_{\Delta}^{2}-3 m_{\pi}^{2}\right)-10 M_{\Delta}^{4}\right]}{D_{\Delta \Delta} \tilde{D}_{\Delta \Delta}^{4}}\right], \\
& f_{\Delta \rightarrow \Delta \pi}^{\text {(end })}(y)=\frac{g_{A}^{2} \bar{\Lambda}^{8}}{50 M_{\Delta}^{2}\left(4 \pi f_{\pi}\right)^{2}} \int d k_{\perp}^{2} \frac{y}{\bar{y}^{2}}\left[\frac{k_{\perp}^{2}+y^{2} M_{\Delta}^{2}+2 \bar{y}^{2}\left(M_{\Delta}^{2}-m_{\pi}^{2}\right)}{\tilde{D}_{\Delta \Delta}^{4}}\right],
\end{aligned}
$$

respectively. The sum of the two terms then gives the total splitting function at $y>0$ as

$$
f_{\Delta \rightarrow \Delta \pi}(y)=\frac{g_{A}^{2} \bar{\Lambda}^{8}}{50 M_{\Delta}^{2}\left(4 \pi f_{\pi}\right)^{2}} \int d k_{\perp}^{2} \frac{y\left[k_{\perp}^{2}+y^{2} M_{\Delta}^{2}\right]}{\bar{y}^{2}} \frac{\left[k_{\perp}^{4}+2 k_{\perp}^{2} M_{\Delta}^{2}\left(\bar{y}+y^{2}\right)+M_{\Delta}^{4}\left(10 \bar{y}^{2}+2 y^{2} \bar{y}^{2}+y^{4}\right)\right]}{D_{\Delta \Delta}^{2} \tilde{D}_{\Delta \Delta}^{4}} .
$$

Finally, for the NLO distribution corresponding to Fig. 1(c), the on-shell and end-point contributions to the $\Delta \rightarrow N \pi$ splitting function are given by

$$
\begin{gathered}
f_{\Delta \rightarrow N \pi}^{\text {(on })}(y)=\frac{g_{A}^{2} \bar{\Lambda}^{8}}{50 M_{\Delta}^{2}\left(4 \pi f_{\pi}\right)^{2}} \int d k_{\perp}^{2} \frac{y\left(\bar{M}^{2}-m_{\pi}^{2}\right)}{\bar{y}^{2}}\left[\frac{\left(\bar{M}^{2}-m_{\pi}^{2}\right)\left(\Delta^{2}-m_{\pi}^{2}\right)}{D_{\Delta N}^{2} \tilde{D}_{\Delta N}^{4}}-\frac{\bar{M}^{2}-3 m_{\pi}^{2}+2 \Delta^{2}}{D_{\Delta N} \tilde{D}_{\Delta N}^{4}}\right], \\
f_{\Delta \rightarrow N \pi}^{\text {end })}(y)=\frac{g_{A}^{2} \bar{\Lambda}^{8}}{50 M_{\Delta}^{2}\left(4 \pi f_{\pi}\right)^{2}} \int d k_{\perp}^{2} \frac{y}{\bar{y}^{2}}\left[\frac{k_{\perp}^{2}+y^{2} M_{\Delta}^{2}-2 y\left(\bar{M}^{2}+M_{\Delta} \Delta\right)-2 \bar{y}^{2} m_{\pi}^{2}-3 \bar{M}^{2}+4 M M_{\Delta}}{\tilde{D}_{\Delta N}^{4}}\right],
\end{gathered}
$$

respectively. The sum of these then gives a total $\Delta \rightarrow N \pi$ splitting function, similar to that in Eq. (9),

$$
f_{\Delta \rightarrow N \pi}(y)=\frac{g_{A}^{2} \bar{\Lambda}^{8}}{50 M_{\Delta}^{2}\left(4 \pi f_{\pi}\right)^{2}} \int d k_{\perp}^{2} \frac{y\left[k_{\perp}^{2}+\left(M-\bar{y} M_{\Delta}\right)^{2}\right]\left[k_{\perp}^{2}+\left(M+\bar{y} M_{\Delta}\right)^{2}\right]^{2}}{\bar{y}^{2} D_{\Delta N}^{2} \tilde{D}_{\Delta N}^{4}} .
$$



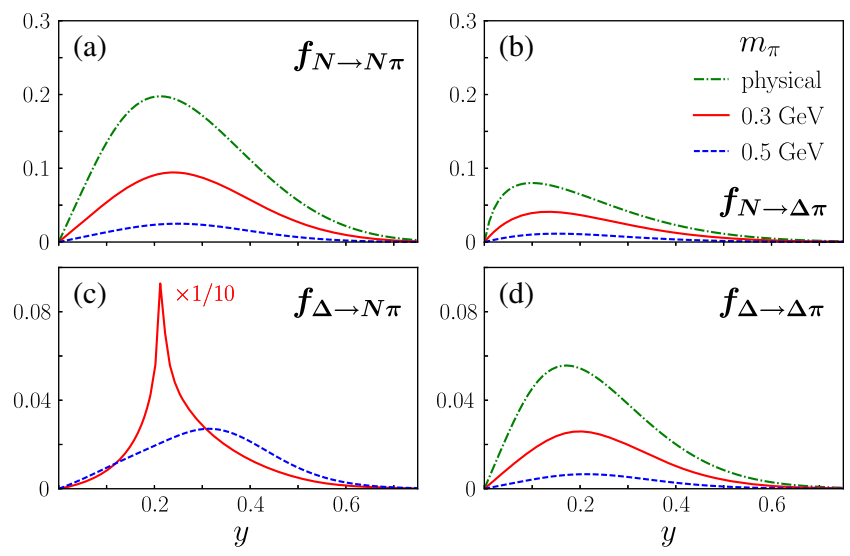

FIG. 2. Chiral splitting functions versus $y$ for the (a) $N \rightarrow N \pi$, (b) $N \rightarrow \Delta \pi$, (c) $\Delta \rightarrow N \pi$, and (d) $\Delta \rightarrow \Delta \pi$ transitions at the physical pion mass (red solid curves), $m_{\pi}=0.3 \mathrm{GeV}$ (blue dashed curves), and $m_{\pi}=0.5 \mathrm{GeV}$ (green dot-dashed curves). Note that the $\Delta \rightarrow N \pi$ function for $m_{\pi}=0.3 \mathrm{GeV}$ has been scaled by a factor $1 / 10$.

In Eqs. (5)-(13), SU(6) symmetry and the GoldbergerTreiman relation have been used to write the $\pi N N, \pi N \Delta$, and $\pi \Delta \Delta$ couplings in terms of the common ratio $g_{A} / f_{\pi}$.

In Fig. 2, we illustrate the four total splitting functions in Eqs. (5), (9), (11), and (13) for a number of values of $m_{\pi}$ relevant for future lattice QCD simulations. The value of the dipole cutoff mass $\Lambda$ was chosen to give an integrated $\bar{d}-\bar{u}$ asymmetry in the proton of 0.1 , which fixes $\Lambda=0.97 \mathrm{GeV}$. The same cutoff value is taken for the $N \Delta \pi$ and $\Delta \Delta \pi$ vertices as for $N N \pi$, and we assume that the cutoff is independent of $m_{\pi}$ (an assumption which is expected to break down at large $m_{\pi}$ ). The nucleon and $\Delta$ masses also depend on $m_{\pi}$, and for these we take the approximate relations $M \approx M^{(0)}+m_{\pi}$ and $M_{\Delta} \approx M_{\Delta}^{(0)}+m_{\pi}$, with the chiral limit values $M^{(0)}=0.8 \mathrm{GeV}$ and $M_{\Delta}^{(0)}=1.1 \mathrm{GeV}$, which have been shown to provide a simple but effective representation of the results of lattice QCD simulations [44].

For the case of the nucleon initial state, the dominance of the LO over the NLO contribution is obvious from Figs. 2(a) and 2(b). The reason is not only the smaller coupling but also the cost in energy to convert the nucleon into a $\Delta$. On the other hand, for a $\Delta$ initial state, the enhancement associated with the exothermic nature of the NLO $\Delta \rightarrow N \pi$ process means that it is larger than the LO $N \rightarrow N \pi$ contribution at all pion masses and is also larger than the $N \rightarrow \Delta \pi$ function.

At $m_{\pi}=0.3 \mathrm{GeV}$, the most prominent feature in the $\Delta \rightarrow N \pi$ splitting function in Fig. 2(b) is the large cusp at $y \approx 0.2$, which indicates the opening of the octet decay channel at $m_{\pi}=\Delta$ (in the present analysis we take the mass difference $\Delta \approx 0.3 \mathrm{GeV}$, independent of $m_{\pi}$ ). Below this threshold the $\Delta \rightarrow N \pi$ function is complex and is not shown in Fig. 2(c) at the physical pion mass. Compared to excited baryon masses, which are found to be relatively

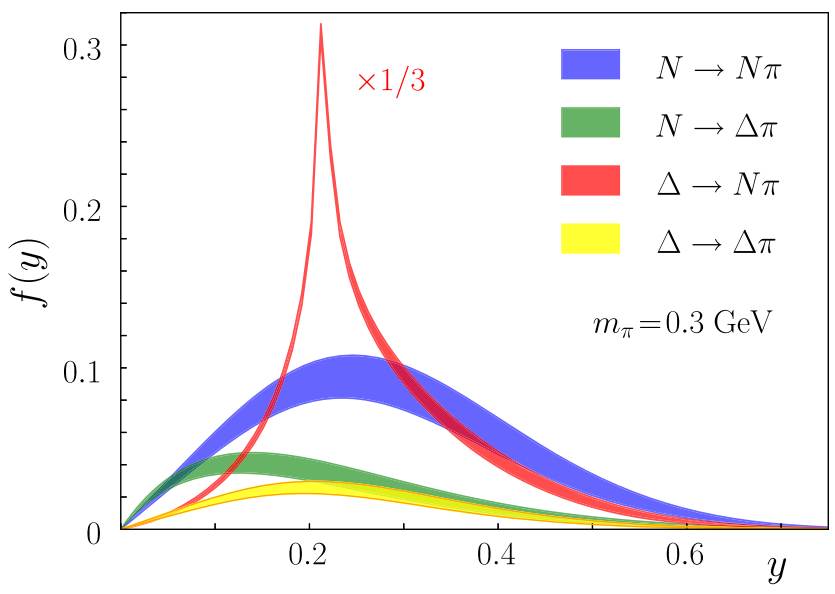

FIG. 3. Chiral splitting functions at $m_{\pi}=0.3 \mathrm{GeV}$, with the shaded bands representing the spread associated with a $\pm 5 \%$ variation of the regulator mass. Note that the $\Delta \rightarrow N \pi$ function has been scaled by a factor of $1 / 3$.

smooth functions of $m_{\pi}$ across the pion decay threshold [36,45], the additional pion propagator in the splitting function enhances the singularity at $m_{\pi} \approx \Delta$ to produce the observed spike. A similar behavior would also be expected for electroweak form factors and indeed was observed in the calculation of pion loop corrections to the $\Delta$ magnetic moments [46].

To make a more direct comparison of the four processes, in Fig. 3 we compare the splitting functions at a fixed value of $m_{\pi}=0.3 \mathrm{GeV}$, at which the differences between the nucleon and $\Delta$ splitting functions are most dramatic. To illustrate the potential dependence on the dipole cutoff mass parameter, $\Lambda$, we indicate the effect of varying $\Lambda$ by $\pm 5 \%$ by the shaded bands. As already indicated in Fig. 2, at this value of $m_{\pi}$ the $\Delta \rightarrow N \pi$ channel dominates, and the presence of the prominent cusp at $y \approx 0.2$ is independent of the choice of regulator. The contributions to the $N$ and $\Delta$ splitting functions from the processes with $\Delta \pi$ intermediate states are significantly smaller than those for the $N \pi$ channels, regardless of the regulator form.

To obtain the $x$ dependence of the $\bar{d}-\bar{u}$ distributions, the splitting functions in Figs. 2 and 3 need to be convoluted with the pion PDF. While the pion valence PDF is relatively well determined from global next-to-leading-order analyses of Drell-Yan and other high energy scattering data [47-49], its dependence on $m_{\pi}$ is less well understood. In the absence of direct lattice calculations of $\bar{q}_{v}^{\pi}$, Detmold et al. [50] used the several low PDF moments from lattice QCD simulations of pion twist-two matrix elements to reconstruct the $x$ dependence over a range of pion masses, from the chiral limit to $m_{\pi}=1 \mathrm{GeV}$, at a scale $Q^{2} \sim 5 \mathrm{GeV}^{2}$, set by the lattice spacing [51].

Using these inputs, in Fig. 4, we show the resulting $\bar{d}-\bar{u}$ asymmetry in the proton and $\Delta^{+}$for several $m_{\pi}$ values 

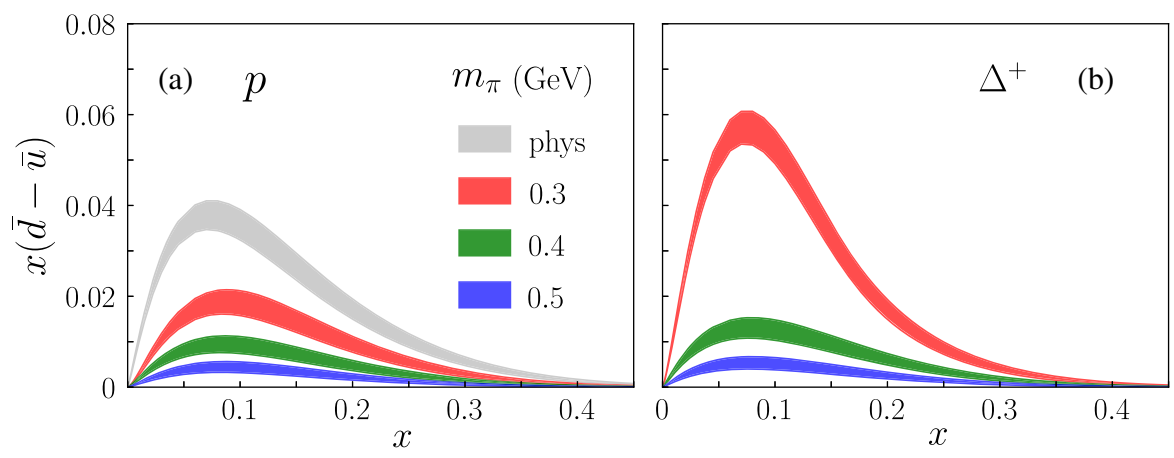

FIG. 4. Predicted $x$ dependence of the $x(\bar{d}-\bar{u})$ asymmetry in (a) the proton and (b) $\Delta^{+}$baryon, for various pion masses: physical $m_{\pi}$ (gray band), $m_{\pi}=0.3 \mathrm{GeV}$ (red), $0.4 \mathrm{GeV}$ (green), and $0.5 \mathrm{GeV}$ (blue). The shaded bands represent the effect of varying the regulator mass parameter $\Lambda$ by $\pm 5 \%$.

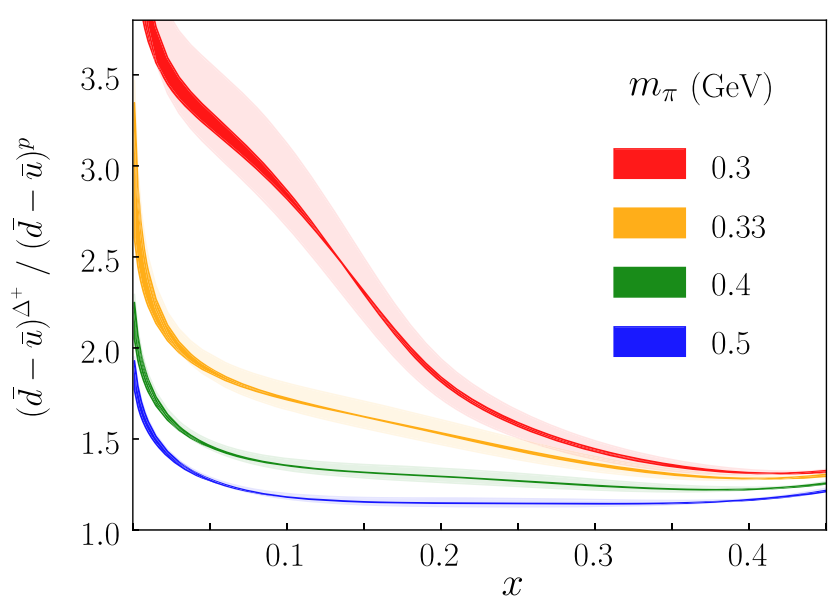

FIG. 5. Ratio of the $\bar{d}-\bar{u}$ asymmetry in the $\Delta^{+}$to that in the proton, for $m_{\pi}=0.3$ (red bands), 0.33 (orange), 0.4 (green), and $0.5 \mathrm{GeV}$ (blue). The darker bands represent the uncertainty on the pion PDF $\bar{q}_{v}^{\pi}$, while the lighter bands represent the dependence on the choice of regulator mass.

ranging from the physical value (for the proton only) to $m_{\pi}=0.5 \mathrm{GeV}$. The bands in Fig. 4 represent uncertainties from the choice of ultraviolet regulator, corresponding to the spread in the splitting functions shown in Fig. 3. While the magnitude of the asymmetry in the proton and $\Delta^{+}$are similar for large values of $m_{\pi} \gtrsim 0.4 \mathrm{GeV}^{2}$, the enhancement due to the opening of the decay channel at $m_{\pi}=\Delta$ renders the asymmetry in the $\Delta^{+}$twice as large near the peak in $x(\bar{d}-\bar{u})$ at $x \approx 0.1$.

The model dependence is expected to cancel to some extent in the ratio of the $\bar{d}-\bar{u}$ asymmetries in the $\Delta^{+}$and $p$, as illustrated in Fig. 5, where the lighter bands show the effect of the $5 \%$ uncertainty in the regulator mass. To highlight the strong enhancement of the $\Delta^{+}$asymmetry as one approaches the $N \pi$ threshold, we compute the ratio at $m_{\pi}=0.3$ and $0.33 \mathrm{GeV}$ [at which $\left(m_{\pi}-\Delta\right) / m_{\pi} \approx 10 \%$ ], in addition to the 0.4 and $0.5 \mathrm{GeV}$ values.
The variation with $m_{\pi}$ is dramatic at $x \approx 0.1$, where the ratio goes from being $\approx 125 \%$ at $m_{\pi}=0.5 \mathrm{GeV}$ to $\approx 300 \%$ just above the threshold at $m_{\pi}=0.3 \mathrm{GeV}$. At larger $x$ values, $x \gtrsim 0.25$, the variation is significantly smaller, resulting in an $\approx 120 \%-140 \%$ enhancement of the $\bar{d}-\bar{u}$ asymmetry across the $m_{\pi}$ values being considered. In this region, the asymmetries are very small, however, and will in practice be difficult to extract from lattice or experiment.

The dependence of the asymmetry ratio on the input pion valence PDF is also relatively weak, as the darker bands in Fig. 5 illustrate. The bands represent the difference between the results using the $m_{\pi}$ dependent pion PDF from Ref. [50] with those using a fixed $\bar{q}_{v}^{\pi}$ PDF at the physical pion mass [52]. Since the same pion PDF enters both the $\Delta^{+}$ and proton convolutions in the numerator and denominator for any $m_{\pi}$, the dependence on $\bar{q}_{v}^{\pi}$ largely cancels, as expected.

The predicted large enhancement of the $\bar{d}-\bar{u}$ asymmetry in the $\Delta^{+}$can be tested in lattice QCD simulations at pion masses just above the $N \pi$ threshold where the $\Delta$ is stable. In particular, the ETM Collaboration plans [53] to calculate the $u-d$ quasi-PDF in the $\Delta$ using the Iwasaki improved gluon action and the twisted mass fermion action with clover improvement [30]. The ensembles to be used in these simulations should allow access to $m_{\pi}$ values at which $\left(m_{\pi}-\Delta\right) / m_{\pi} \approx 3 \%$ [24], which could provide a striking confirmation of the role of chiral symmetry and the pion cloud in the generation of a nonperturbative sea in baryons.

\section{ACKNOWLEDGMENTS}

This work was supported by the University of Adelaide and by the Australian Research Council through the ARC Centre of Excellence for Particle Physics at the Terascale (CE110001104) and Discovery Project DP150103164, and the U.S. Department of Energy Award No. DE-AC0506OR23177, under which Jefferson Science Associates, LLC operates Jefferson Lab. F. S. was funded by DFG Project No. 392578569. J. J. E. was partially supported by the Netherlands Organization for Scientific Research. 
[1] P. Amaudruz et al., Phys. Rev. Lett. 66, 2712 (1991).

[2] K. Gottfried, Phys. Rev. Lett. 18, 1174 (1967).

[3] A. W. Thomas, Adv. Nucl. Phys. 13, 1 (1984).

[4] S. Theberge, A. W. Thomas, and G. A. Miller, Phys. Rev. D 22, 2838 (1980); 23, 2106(E) (1981).

[5] A. W. Thomas, Phys. Lett. 126B, 97 (1983).

[6] W. Melnitchouk, A. W. Thomas, and A. I. Signal, Z. Phys. A 340, 85 (1991).

[7] S. Kumano and J. T. Londergan, Phys. Rev. D 44, 717 (1991).

[8] E. M. Henley and G. A. Miller, Phys. Lett. B 251, 453 (1990).

[9] J. Speth and A. W. Thomas, Adv. Nucl. Phys. 24, 83 (1997).

[10] A. W. Thomas, W. Melnitchouk, and F. M. Steffens, Phys. Rev. Lett. 85, 2892 (2000).

[11] W. Detmold, W. Melnitchouk, J. W. Negele, D. B. Renner, and A. W. Thomas, Phys. Rev. Lett. 87, 172001 (2001).

[12] J. W. Chen and X. Ji, Phys. Lett. B 523, 73 (2001).

[13] D. Arndt and M. J. Savage, Nucl. Phys. A697, 429 (2002).

[14] J. W. Chen and X. Ji, Phys. Rev. Lett. 88, 052003 (2002).

[15] A. Baldit et al., Phys. Lett. B 332, 244 (1994).

[16] E. A. Hawker et al., Phys. Rev. Lett. 80, 3715 (1998).

[17] W. Melnitchouk, J. Speth, and A. W. Thomas, Phys. Rev. D 59, 014033 (1998).

[18] Fermilab E906 Experiment (SeaQuest), Drell-Yan measurements of nucleon and nuclear structure with the Fermilab main injector, http://www.phy.anl.gov/mep/SeaQuest/index .html.

[19] X. Ji, Phys. Rev. Lett. 110, 262002 (2013).

[20] C. Alexandrou, K. Cichy, M. Constantinou, K. Hadjiyiannakou, K. Jansen, H. Panagopoulos, and F. Steffens, Nucl. Phys. B923, 394 (2017).

[21] J. W. Chen, T. Ishikawa, L. Jin, H.-W. Lin, Y. B. Yang, J. H. Zhang, and Y. Zhao, Phys. Rev. D 97, 014505 (2018).

[22] J. Green, K. Jansen, and F. Steffens, Phys. Rev. Lett. 121, 022004 (2018).

[23] X. Xiong, X. Ji, J. H. Zhang, and Y. Zhao, Phys. Rev. D 90, 014051 (2014).

[24] C. Alexandrou, K. Cichy, V. Drach, E. Garcia-Ramos, K. Hadjiyiannakou, K. Jansen, F. Steffens, and C. Wiese, Phys. Rev. D 92, 014502 (2015).

[25] T. Izubuchi, X. Ji, L. Jin, I. W. Stewart, and Y. Zhao, Phys. Rev. D 98, 056004 (2018).

[26] W. Detmold, W. Melnitchouk, and A. W. Thomas, Mod. Phys. Lett. A 18, 2681 (2003).

[27] H.-W. Lin, J.-W. Chen, S. D. Cohen, and X. Ji, Phys. Rev. D 91, 054510 (2015).

[28] J.-W. Chen, S. D. Cohen, X. Ji, H.-W. Lin, and J.-H. Zhang, Nucl. Phys. B911, 246 (2016).

[29] C. Alexandrou, K. Cichy, M. Constantinou, K. Hadjiyiannakou, K. Jansen, F. Steffens, and C. Wiese, Phys. Rev. D 96, 014513 (2017).
[30] C. Alexandrou, K. Cichy, M. Constantinou, K. Jansen, A. Scapellato, and F. Steffens, Phys. Rev. Lett. 121, 112001 (2018).

[31] J. W. Chen, L. Jin, H.-W. Lin, Y. S. Liu, Y. B. Yang, J. H. Zhang, and Y. Zhao, arXiv:1803.04393.

[32] X. G. Wang, C.-R. Ji, W. Melnitchouk, Y. Salamu, A. W. Thomas, and P. Wang, Phys. Rev. D 94, 094035 (2016).

[33] Y. Salamu, C. R. Ji, W. Melnitchouk, and P. Wang, Phys. Rev. Lett. 114, 122001 (2015).

[34] M. Burkardt, K. S. Hendricks, C. R. Ji, W. Melnitchouk, and A. W. Thomas, Phys. Rev. D 87, 056009 (2013).

[35] C. R. Ji, W. Melnitchouk, and A. W. Thomas, Phys. Rev. D 88, 076005 (2013).

[36] R. D. Young, D. B. Leinweber, and A. W. Thomas, Prog. Part. Nucl. Phys. 50, 399 (2003).

[37] B. Z. Kopeliovich, I. K. Potashnikova, B. Povh, and I. Schmidt, Phys. Rev. D 85, 114025 (2012).

[38] J. R. McKenney, N. Sato, W. Melnitchouk, and C.-R. Ji, Phys. Rev. D 93, 054011 (2016).

[39] A. W. Thomas, Nucl. Phys. B, Proc. Suppl. 119, 50 (2003).

[40] J. M. M. Hall, D. B. Leinweber, and R. D. Young, Phys. Rev. D 82, 034010 (2010).

[41] Y. Salamu, C. R. Ji, W. Melnitchouk, A. W. Thomas, and P. Wang, Phys. Rev. D 99, 014041 (2019).

[42] S. D. Drell, D. J. Levy, and T. M. Yan, Phys. Rev. D 1, 1035 (1970).

[43] A. W. Thomas and G. Krein, Phys. Lett. B 481, 21 (2000).

[44] A. Walker-Loud, Proc. Sci., LATTICE2008 (2008) 005.

[45] D. B. Leinweber, A. W. Thomas, K. Tsushima, and S. V. Wright, Phys. Rev. D 61, 074502 (2000).

[46] I. C. Cloët, D. B. Leinweber, and A. W. Thomas, Phys. Lett. B 563, 157 (2003).

[47] P. C. Barry, N. Sato, W. Melnitchouk, and C.-R. Ji, Phys. Rev. Lett. 121, 152001 (2018).

[48] P. J. Sutton, A. D. Martin, W. J. Stirling, and R. G. Roberts, Phys. Rev. D 45, 2349 (1992).

[49] M. Aicher, A. Schäfer, and W. Vogelsang, Phys. Rev. Lett. 105, 252003 (2010).

[50] W. Detmold, W. Melnitchouk, and A. W. Thomas, Phys. Rev. D 68, 034025 (2003).

[51] C. Best, M. Göckeler, R. Horsley, E.-M. Ilgenfritz, H. Perlt, P. Rakow, A. Schäfer, G. Schierholz, A. Schiller, and S. Schramm, Phys. Rev. D 56, 2743 (1997).

[52] M. Oehm, M. Oehm, C. Alexandrou, M. Constantinou, K. Jansen, G. Koutsou, B. Kostrzewa, F. Steffens, C. Urbach, and S. Zafeiropoulos (ETM Collaboration), Phys. Rev. D 99, 014508 (2019).

[53] F. M. Steffens, C. Alexandrou, S. Bacchio, K. Cichy et al., $\Delta$-baryon parton distribution functions from lattice QCD, Project Proposal for Tier 0/Tier 1 HPC Access, Gauss Centre for Supercomputing. 